\title{
Assessment of comprehensive HIV-risk reduction programming for adolescent girls and young women: Implementation science research in Kenya
}

Population Council

Follow this and additional works at: https://knowledgecommons.popcouncil.org/departments_sbsr-hiv

Part of the Demography, Population, and Ecology Commons, Family, Life Course, and Society Commons, International Public Health Commons, and the Medicine and Health Commons How does access to this work benefit you? Let us know!

\section{Recommended Citation}

Population Council. 2017. "Assessment of comprehensive HIV-risk reduction programming for adolescent girls and young women: Implementation science research in Kenya," Activity brief. Washington, DC: Population Council. 


\section{ASSESSMENT OF COMPREHENSIVE HIV-RISK REDUCTION PROGRAMMING FOR ADOLESCENT GIRLS AND YOUNG WOMEN

HIV prevalence in Kenya is estimated to be 5.6 percent. Yet the proportion of the population who are HIV positive varies significantly by region, with prevalence as high as 18.7 percent in Kisumu County-the site of the current study. Adolescent girls and young women (AGYW) aged 15-24 have higher odds of being infected by HIV compared to men of the same age group. ${ }^{1}$ Further, there are indications that HIV prevalence has increased substantially among youth (aged 15-24 years) in recent years. $^{2}$

AGYW's vulnerability in Kenya, as in many countries, is due to a host of reasons, including limited knowledge about HIV, low risk perception, and high levels of risk behaviors. Broader social factors typically include early marriage, poverty, gender inequality, sexual violence, lack of education, unemployment, and unequal power relations with older male partners. Further, programmatic factors include limited access to and utilization of health services, resulting from health provider's attitudes, inconvenient clinic hours or locations, cost of services, and health care staff inadequately trained to deal with adolescent health needs.

In collaboration with the Kenyan government, the PEPFAR-supported DREAMS (Determined, Resilient, Empowered AIDS-free, Mentored, and Safe) Partnership in Kenya is responding to these challenges by delivering through existing platforms a comprehensive package of interventions designed to empower AGYW, mobilize communities, strengthen families, and reduce AGYW's sexual risk. Trained providers also are offering youth friendly services or making appropriate referrals based on individual AGYW needs.

The Population Council is conducting implementation research to build needed evidence to inform decision-making around effective implementation of community-based, girl-centred interventions and to assess their effectiveness in reducing HIV vulnerability among AGYW-a population in which AIDS is the leading cause of death in the region.

Learnings from this study and similar ones in Zambia and Malawi will be valuable for informing each country and the region how to implement AGYW programming that goes beyond the health sector to reduce AGYW's vulnerability to HIV.

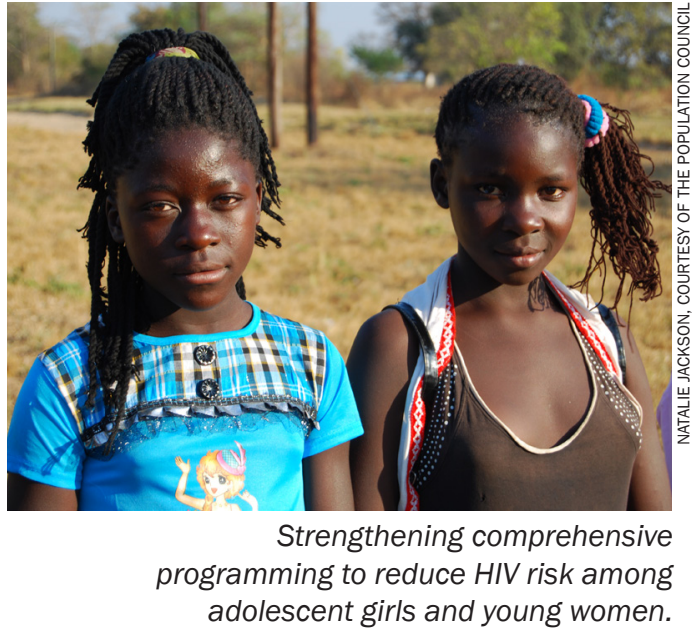

Location: Nyalenda and Kolwa East in Kisumu County

Study Duration: 2016-2018

Funder: Bill \& Melinda Gates Foundation

For more information, contact Sanyukta Mathur (smathur@ popcouncil.org), or Jerry Okal (jokal@ popcouncil.org). 


\section{POPULATION COUNCIL'S DREAMS IMPLEMENTATION RESEARCH}

This study is part of a portfolio of implementation science studies the Population Council is conducting, with funding from the Bill \& Melinda Gates Foundation, related to the DREAMS Partnership. This Partnership aims to significantly reduce new HIV infections among AGYW in 10 countries in subSaharan Africa, including Kenya. Our studies are exploring whether the intended beneficiaries-AGYW and their male partners-are being reached and retained in services, how the interventions are being delivered to each group, and ultimately whether HIV risk among AGYW is being reduced.

\section{OUR RESEARCH}

We are using a mixed-method research design and gathering data with AGYW and program implementers. We conducted a community-based household survey with AGYW ( $n=864$ ) and a survey with AGYW who are enrolled in DREAMS programming ( $n=914)$ to compare and assess if the most vulnerable AGYW were identifed and reached. We will conduct a 12-month follow-up survey with the cohort of AGYW enrolled in the program to examine program engagement and HIV risk reduction. Additionally, we are collecting qualitative data from a sub-group of AGYW ( $n=36$ ) during the course of the study to examine their motivations for participating in DREAMS programs, program experiences, and use of program services.

\section{Using direct observations and interviews with} implementing partners $(n=24)$ we will assess facilitators and barriers to programming. Finally, in collaboration with the implementing partner, we will assess the costs associated with the DREAMS core package of interventions in the study sites.

The study will provide critical information to policymakers and programmers in designing relevant girl-centred, HIVrisk reduction programs in Kenya and sub-Saharan Africa.

\section{RESEARCH UTILIZATION}

We will actively engage key stakeholders throughout the study, including gaining their insights on key study questions, interpreting the findings, crafting initial programming recommendations based on findings, and supporting their adoption in Kenya and beyond. Stakeholders in Kenya including the Ministry of Health, National AIDS \& STI Control Programme, National AIDS Control Council, PEPFAR-Kenya, PATH/APHIAPIus and other DREAMS implementing partners, county health departments, county AIDS and STI coordinators, youth and AIDS service organizations, and media representatives.

\section{WHAT WILL WE LEARN?}

This innovative implementation science study will generate valuable evidence about the effectiveness of programs designed to respond to the individual, social, and health service-related factors that determine AGYW's vulnerability to HIV. Specifically the study will:

- Assess the ability of DREAMS programs to identify and engage the most at-risk AGYW.

- Determine the effectiveness of the DREAMS interventions to reduce HIV risk among AGYW participants.

- Identify major barriers to and facilitators of successful implementation of community-based, girl-centered HIV programming.

- Determine program costs and resources associated with delivering a comprehensive intervention package to reduce HIV risk among AGYW.

${ }^{1}$ National AIDS Control Council. 2014. Kenya AIDS Response Progress Report 2014 Progress towards Zero. Nairobi:

${ }^{2}$ Odhiambo ,R. 2016. "46\% of new HIV infections among adolescents and young people," The Star, Kenya, Apr. 20.
POPULATION COUNCIL

Ideas. Evidence. Impact.
The Population Council confronts critical health and development issues-from stopping the spread of HIV to improving reproductive health and ensuring that young people lead full and productive lives. Through biomedical, social science and public health research in about 50 countries, the Council works with our partners to deliver solutions that lead to more effective policies, programs, and technologies to improve lives worldwide. Established in 1952 and headquartered in New York, the Council is a nongovernmental, nonprofit organization with an international board of trustees. popcouncil.org

Suggested citation: Population Council. 2017. "Assessment of comprehensive HIV-risk reduction programming for adolescent girls and young women: implementation science research in Kenya," DREAMS Activity Brief. Washington, DC: Population Council. 